\title{
Robust Image Watermarking Scheme Using Population-Based Stochastic Optimization Technique
}

\author{
Tamirat Tagesse Takore \\ Department of Electronics and Communication Engineering, Andhra University, Visakhapatnam, India \\ Email: tameauce2014@gmail.com \\ ${ }^{\text {a } P . ~ R a j e s h ~ K u m a r ~ a n d ~}{ }^{\mathrm{b}}$ G. Lavanya Devi \\ ${ }^{\text {a } P r o f . ~ i n ~ D e p ' t . ~ o f ~ E l e c t r o n i c s ~ a n d ~ C o m m u n i c a t i o n ~ E n g i n e e r i n g, ~}{ }^{\mathrm{b}}$ Ass. Prof. in Dep't. of Computer Science \& System \\ Engineering, Andhra University, Visakhapatnam, India \\ Email: rajeshauce@gmail.com, lavanyadevig@yahoo.co.in
}

\begin{abstract}
Designing an efficient watermarking scheme that can achieve better robustness with limited visual quality distortion is the most challenging problem. In this paper, robust digital image watermarking scheme based on edge detection and singular value decomposition (SVD) is proposed. Two sub-images, which are used as a point of reference for both watermark embedding and extracting, are formed from blocks that are selected based on the number of edges they have. Block based SVD is performed on sub-images to embed a binary watermark by modifying the singular value (S). A population-based stochastic optimization technique is employed to achieve enhanced performance by searching embedding parameters which can maintain a better trade-off between robustness and imperceptibility. The experimental results show that the proposed method achieves improved robustness against different image processing and geometric attacks for selected quality threshold. The performance of the proposed scheme is compared with the existing schemes and significant improvement is observed.
\end{abstract}

Index Terms-Image watermarking, edge detection, singular value decomposition, particle swarm optimization, multiple scaling factors, PSNR, BCR.

\section{INTRODUCTION}

The advancement and widespread use of digital computers along with high-speed networks make digitally formatted multimedia data such as text, image, video, and audio easy to be copied, modified and shared. Malicious attackers can take this as an opportunity and use the technology to misuse the valuable digital resources of others by violating intellectual property right. Hence, the need for effective copyright protecting tools is growing from time to time and become the critical issue for content producers and publishers. Different data securing techniques have been proposed by numerous researchers to address the growing concern. Among these techniques, digital watermarking is one of the most popular ways to solve multimedia data copyright and content authentication problems.

Digital watermarking is a process that embeds a watermark also known as a digital signature within a host multimedia object such that the embedded watermark, which completely characterizes the person who applies it, can be extracted later at the time of dispute to make an assertion about the object. In addition to copyright protection and content authentication, digital watermarking can be used for various applications such as copy control, broadcast monitoring, annotation and content description, ownership identification, tamper detection and covert communication [1,2].

Digital watermarking techniques can be classified into various categories in numerous ways [3]. Based on the embedding domain, they can be categorized into spatial domain and transform domain watermarking techniques. Spatial domain watermarking techniques embed watermarks into multimedia objects directly modifying selected pixels intensity values. Usually, these techniques are computationally simple and easy to implement [4]. On the other hand, transform domain watermarking techniques insert the watermarks by modifying values of transform coefficients of cover objects [5]. The most common transforming techniques such as Discrete Cosine Transform (DCT), Discrete Fourier Transform (DFT), Discrete Wavelet Transform (DWT) and Singular Valued Decomposition (SVD) have been used in transform domain watermarking algorithms. Transform domain watermarking techniques provide more robust and imperceptible watermarked data than spatial domain watermarking techniques [6,7].

Furthermore, depending upon the type of data to be watermarked, the watermarking methods can be classified into four categories: text watermarking, image watermarking, audio watermarking, and video watermarking. However, this paper presents a robust grayscale image watermarking scheme.

Imperceptibility and robustness are the two desired watermarking properties which often use as a criterion to 
evaluate the effectiveness of watermarking schemes. The term imperceptibility refers to the degree of visual similarity between the original unwatermarked and watermarked images. In fact, watermark inserting process can degrade the quality of host image; however, for an invisible watermarking scheme, the embedding process should maintain the visual quality in acceptable level so that the modification made to host image cannot be noticed easily by an observer. A watermarking scheme is said to be robust if it gives a watermarked document that unauthorized party can not destroy the embedded information without also making the watermarked document useless. The embedded watermark withstands common signal processing attacks and can be extracted or detected even after the attacks.

The rest of this paper is organized as follows. In section II, a brief review of related work is presented. Section III presents feature based reference sub-image forming technique. Detail of SVD is discussed in section IV. Section V illustrates the proposed watermark embedding and extraction procedure. Section VI presents experimental setup and results. Finally, Section VII ends the paper with a conclusion.

\section{RELATED WORKS}

Designing robust digital image watermarking schemes, which can embed watermarks without significantly affecting the visual quality of the host images, have become the major challenge in the field of digital watermarking due to the existence of two conflicting watermarking requirements, i.e., robustness and imperceptibility. Researchers across the globe have proposed various watermarking schemes using different techniques, both in spatial and transform domains, aiming to address the problem. They have also employed different optimization techniques, which mimic biological phenomena, to improve the performance of the scheme by searching optimal parameters for watermark embedding.

Because of its unique features, SVD has become the most popular mathematical method for watermark embedding, and numerous robust image watermarking schemes have been proposed using it. A new binary watermark embedding algorithm based on chaotic mixing and SVD for copyright protection application was proposed in [8]. The proposed method embeds a mixed binary watermark into the left orthogonal matrix of the host image. The main limitation of this work is the level of robustness is low for various attacks. Authors in [9] proposed SVD based noble watermarking algorithm which can perform well in both robustness and security for ownership protection applications. They have used a grayscale image, and the algorithm has been tested using six practical conditions, i.e., adding noise, low-pass filtering, JPEG compression, scaling, image cropping, and rotation. However, the method, which they have used to select scaling factor for coefficient modification, will not ensure the trade-off between the robustness and imperceptibility. Using optimization techniques can improve the performance of the algorithm by searching best embedding parameters which can ensure a balance between imperceptibility and robustness. In [10] an evolutionary based digital image watermarking algorithm using SVD technique was proposed. The original image is divided into $8 \times 8$ blocks to insert a binary watermark within singular value using quantization method. The performance of proposed algorithm has been improved by employing a genetic algorithm to generate binary watermark. Authors in [11] employed particle swarm optimization (PSO) and wavelet domain technique to propose an intelligent watermarking scheme which can overcome the security problem. The strength of embedding watermark in different blocks is controlled by invoking a PSO method to resolve the conflict between imperceptibility and robustness. But, it is also worthy to take into consideration that both imperceptibility and robustness highly depend on the methods employed to select regions or blocks of the images for watermark embedding. In recent years, feature-based image watermarking schemes that embed desired watermark into selected regions with features like edge, texture or fractal dimension, have been introduced. Selecting different regions of the image with different features may depend on the watermarking applications and directly influences the level of robustness and imperceptibility of the scheme. In [12] a robust image watermarking algorithm, which can resist both geometric distortion and signal processing attacks, was proposed using a feature extraction method called Mexican Hat wavelet scale interaction. The authors have used the extracted feature as reference points for both watermark embedding and detection. Authors in [13] proposed a robust, featurebased image watermarking scheme using discrete wavelet transform and singular value decomposition techniques. The PSO algorithm offers a systematic way to consider the improvements of the scaling factor that controls the strength of the embedded watermark.

Even though several image watermarking algorithms for different application have been proposed so far, conducting further research in the field is still required to fill existing gaps. In this paper, an optimized grayscale image watermarking scheme using block based SVD, edge detection and PSO technique is proposed. Since human eyes are less sensitive to changes take place in regions of the image having more number of edge or texture, embedding watermark in these regions may not degrade the quality significantly. Hence, in our work Canny edge detector is employed to select blocks with large number of edge pixels to embed a binary watermark. The particle swarm optimization (PSO) algorithm is employed to search appropriate scaling factors which determine the embedding strength of a watermark.

\section{FEATURE-BASED REFERENCE IMAGE FORMATION}

Feature-based digital image watermarking techniques have become the most popular strategy to design robust and imperceptible watermarking scheme. The existence of various feature detection techniques enables designers 
to select different regions or blocks of an image for watermark embedding. Various feature detectors extract local feature by performing specific transformations on images. In digital watermarking, regions or blocks having desired features can be used as a reference point for both watermark embedding and extraction. In this work, we have used the canny edge detector for selecting image blocks to embed a binary watermark. Even though there are number of edge detecting techniques, we have preferred canny edge detector due to its superior performance over the others. First, original image IG of size $\mathrm{M} \times \mathrm{N}$ is partitioned into non-overlapping blocks $\mathrm{Bk}$ of size $m \times n$. Then, edges are extracted from each block using canny detector, and total number edge pixels in the block $\left(\mathrm{N}_{\mathrm{k}}^{\mathrm{E}}\right.$ ) are computed using the following equations.

$$
\begin{gathered}
E_{k}=\operatorname{edge}\left(B_{k}\right), \text { for } k=1,2, \ldots, n_{b} \\
N_{k}^{E}=\sum_{i=1}^{m} \sum_{j=1}^{n} E_{k}(i, j)
\end{gathered}
$$

where, edge (.) represents the edge detection operation, $\mathrm{n}_{\mathrm{b}}$ is the total number of blocks, $E_{k}(i, j)=1$ for edge pixels and 0 otherwise. The blocks are reordered based on the number of edges keeping their position as a key $\left(Q_{k}\right)$ to use it later. To embed watermark of size $\mathrm{p} \times \mathrm{q}$, a total of $\mathrm{N}_{\mathrm{t}}$ (where, $\mathrm{N}_{\mathrm{t}}=\mathrm{pq}$ ) numbers of blocks are required and the first reference image $\mathrm{I}_{\mathrm{rf}}^{\mathrm{H}}$ is formed by merging these blocks together. The same numbers of blocks with the least number of edge pixels are also selected to form the second reference image $\mathrm{I}_{\mathrm{rf}}^{\mathrm{L}}$, which uses as a reference image for watermark embedding and extraction.

$$
\begin{gathered}
I_{r f}^{H}(\mathrm{p}, \mathrm{q})=\bigcup_{k=1}^{N_{t}}(B)_{k}^{H} \\
I_{r f}^{L}(\mathrm{p}, \mathrm{q})=\bigcup_{k=1}^{N_{t}}(B)_{k}^{L}
\end{gathered}
$$

Where, $\mathrm{U}($.$) represents blocks merging operation, B_{k}^{H}$ and $B_{k}^{L}$ are blocks with large and small number of edges respectively. In Fig. 1 the overall steps involved in the reference image formation are depicted well.

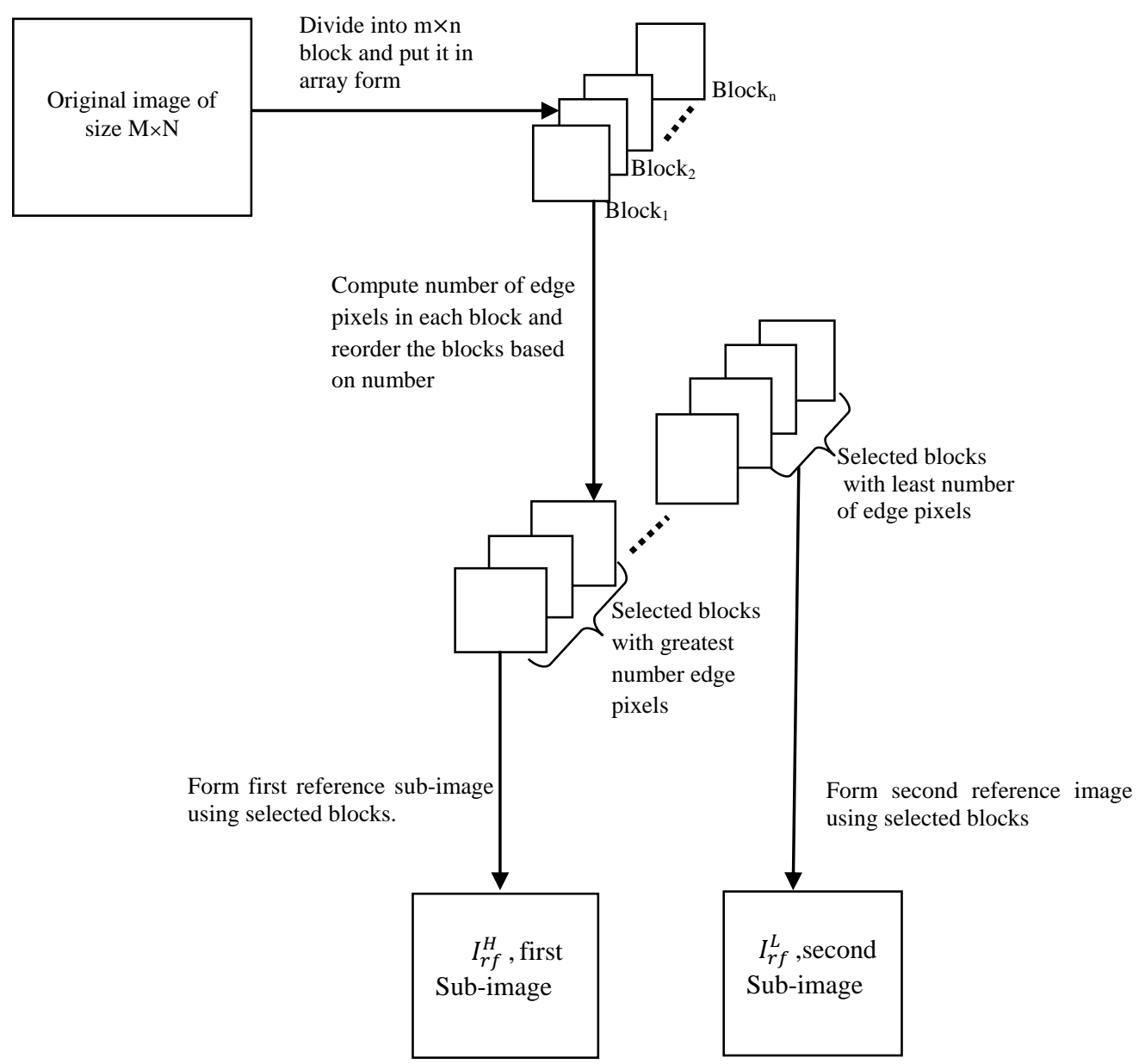

Fig.1. Flow chart for reference sub-images formation. 


\section{Singular Value DeComposition}

Singular value decomposition is linear algebra technique, which uses to factorize a real or complex rectangular matrix into three unique matrices. If $\mathrm{Y}$ is a real M-by-N matrix, then there exist two orthogonal matrices $\mathrm{U}$ and $\mathrm{V}$ (i.e., $\mathrm{UU}^{\mathrm{T}}=\mathrm{I}, \mathrm{VV}^{\mathrm{T}}=\mathrm{I}$ ) and one rectangular diagonal matrix $S$, such that

$$
Y=U S V^{T}=\left(\begin{array}{ccc}
u_{11} & \cdots & u_{1 M} \\
u_{21} & \ldots & u_{2 M} \\
\vdots & \ddots & \vdots \\
u_{M 1} & \ldots & u_{M M}
\end{array}\right)\left(\begin{array}{cccc}
s_{11} & 0 & \ldots & 0 \\
0 & s_{22} & & \\
\vdots & \vdots & \ddots & \vdots \\
0 & 0 & \ldots & s_{M N}
\end{array}\right)\left(\begin{array}{ccc}
v_{11} & \ldots & v_{1 N} \\
v_{21} & \ldots & v_{2 M} \\
\vdots & \ddots & \vdots \\
v_{N 1} & \ldots & v_{N N}
\end{array}\right)^{T}
$$

where matrix $\mathrm{S}$ is a nonnegative diagonal matrix containing the square roots of the eigenvalues of either $\mathrm{U}$ or $\mathrm{V}$ arranged in descending order diagonally (i.e., $\mathrm{s}_{11} \geq$ $\mathrm{s}_{22} \geq \mathrm{s}_{33} \geq \ldots \geq \mathrm{s}_{\mathrm{MN}}$ ). SVD has great application in the field of image processing, especially in digital image watermarking. The followings are some of the advantages that we can get from SVD when we use it in a watermarking algorithm.

i. Image quality is not affected significantly due to a slight modification of singular values during watermark embedding process.

ii. Singular values have good stability and their values are rotationally invariant.

iii. SVD can pack large energy signal within few coefficients.

In this paper, we have used SVD to factorize the subimages to embed a binary watermark.

\section{PROPOSED SCHEME}

Using multiple scaling factors (MSF) other than a single scaling factor (SSF) to modulate different ranges of selected coefficients during watermark embedding process can significantly improve the performance of the scheme. In this work, block based SVD is performed on reference sub-images ( $I_{r f}^{H}$ and $I_{r f}^{L}$ ) to obtain the respective singular value coefficients $\left(S_{r f(p, q)}^{H}\right.$ and $\left.S_{r f(p, q)}^{L}\right)$. Then the difference $\left(\mathrm{D}_{(\mathrm{p}, \mathrm{q})}\right)$ between $S_{r f(p, q)}^{H}$ and $S_{r f(p, q)}^{L}$ is computed using the following equation:

$$
D(p, q)=\left|S_{r f(p, q)}^{H}(i, j)-S_{r f(p, q)}^{L}(i, j)\right|
$$

where $\mathrm{i}=\mathrm{j}=1$ and $(\mathrm{p}, \mathrm{q})$ refer to block indices. $\mathrm{D}_{(\mathrm{p}, \mathrm{q})}$ values are again divided into $\mathrm{n}$ number of non-overlapping sub ranges $\left(\mathrm{r}_{\mathrm{k}}\right)$ so that multiple scaling factors $\left(\beta_{\mathrm{k}}\right)$ can be used to embed a watermark based on the magnitude of $\mathrm{D}_{(\mathrm{p}, \mathrm{q})}$.

$$
\left.\begin{array}{l}
D_{\max }=\max (D(p, q)) \\
D_{\min }=\min (D(p, q)) \\
d=\left(D_{\max }-D_{\min }\right) / n \\
r_{k}=[(k-1) d,(k d)), \text { fork }=1,2, \ldots, n
\end{array}\right\}
$$

Therefore, for the completion of embedding process $n$ numbers of scaling factors are required, and in this paper, we have used five scaling factors $\left(\beta_{\mathrm{k}}\right)$, where $\mathrm{k}=1,2 \ldots 5$ such that $\beta_{\mathrm{k}}>\beta_{\mathrm{k}+1}$. After obtaining each sub-range interval, a singular value coefficient that belongs to a particular sub-range are modified by a corresponding scaling factor depending on watermark bits as per equation (8).

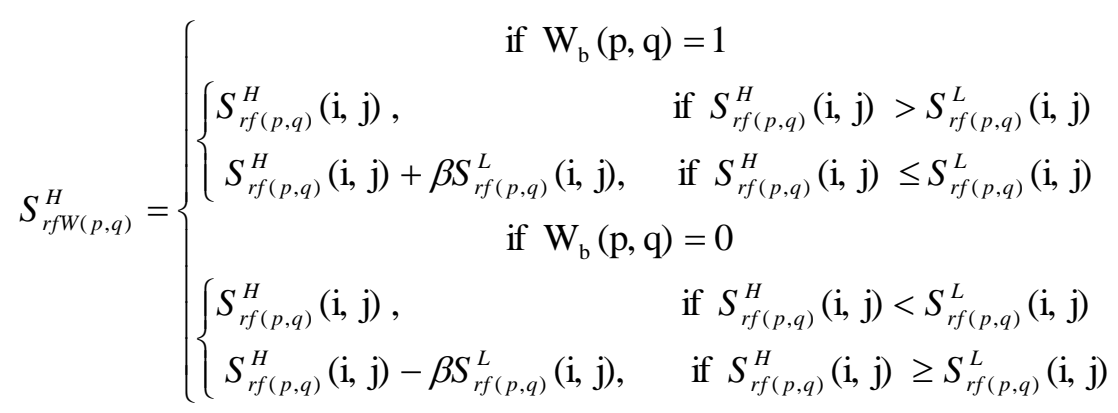

where scaling factor $\beta=\beta_{k}$ if $D_{(p, q)}$ is in the range $r_{k}$ and $\mathrm{i}=\mathrm{j}=1$.

\section{A. Watermark Embedding Technique}

Watermark embedding algorithm takes original host image $\mathrm{I}_{\mathrm{G}}$ and binary watermark $\mathrm{W}_{\mathrm{b}}$ of sizes $\mathrm{M} \times \mathrm{N}$ and $\mathrm{p} \times \mathrm{q}$ respectively as input and provide watermarked image $I_{w}$ as an output. The followings are the steps for the proposed watermark embedding algorithm.

Step1: Original host image $\mathrm{I}_{\mathrm{G}}$ is decomposed into two references sub-images $I_{r f}^{H}$ and $I_{r f}^{L}$ using the procedure discussed in section III. 
Step2: Perform block based SVD operation on $I_{r f}^{H}$ and $I_{r f}^{L}$.

$$
\left.\begin{array}{l}
{\left[U_{r f(p, q)}^{H}, S_{r f(p, q)}^{H}, V_{r f(p, q)}^{H}\right]=S V D\left(I_{r f(p, q)}^{H}\right)} \\
{\left[U_{r f(p, q)}^{L}, S_{r f(p, q)}^{L}, V_{r f(p, q)}^{L}\right]=S V D\left(I_{r f(p, q)}^{L}\right)}
\end{array}\right\}
$$

Step3: Embed binary watermark $\mathrm{W}_{\mathrm{b}}(\mathrm{p}, \mathrm{q})$ by modifying $S_{r f(p, q)}^{H}$ based on the value of the watermark bits as per equation (8) and use PSO based procedure to find best scaling factors $\left(\beta_{k}\right)$ for each sub-range.

Step4: Apply inverse SVD operation to obtain watermarked blocks $\mathrm{B}_{\mathrm{w}}^{\mathrm{H}}$.

$$
B_{W(p, q)}^{H}=U_{r f(p, q)}^{H} S_{r f W(p, q)}^{H} V_{r f(p, q)}^{H^{T}}
$$

Step5: Place each block to their original position using key $\mathrm{Q}_{\mathrm{k}}$ to obtain watermarked image $\mathrm{I}_{\mathrm{w}}$.

\section{B. Watermark Extraction Technique}

Possibly an attacked watermarked image $\mathrm{I}_{\mathrm{W}}^{*}$ and key $\left(\mathrm{Q}_{\mathrm{k}}\right)$ are required as inputs for the extraction process. The steps for watermark extraction are given as follows.

Step1: Decompose possibly attacked watermarked image into two sub-images $\left(I_{r f w^{H^{*}}}^{H^{*}}\right.$ and $\left.I_{r f w}^{L^{*}}\right)$ using key and procedure discussed in section III.

Steps2: Apply block based SVD on $I_{r f w}^{H^{*}}$ and $I_{r f w}^{L^{*}}$.

$$
\left.\begin{array}{l}
{\left[U_{w(p, q)}^{H^{*}}, S_{w(p, q)}^{H^{*}}, V_{w(p, q)}^{H^{*}}\right]=S V D\left(I_{r f w(p, q)}^{H^{*}}\right)} \\
{\left[U_{w(p, q)}^{L^{*}}, S_{w(p, q)}^{L^{*}}, V_{w(p, q)}^{L^{*}}\right]=S V D\left(I_{r f w(o, q)}^{L}\right)}
\end{array}\right\}
$$

Steps3: Extract the watermark using the rule given in equation (12).

$$
W_{E}(p, q)= \begin{cases}1, & \text { if } S_{w(p, q)}^{H^{*}}(\mathrm{i}, \mathrm{j}) \geq S_{w(p, q)}^{L^{*}}(\mathrm{i}, \mathrm{j}) \\ 0, & \text { otherwise. }\end{cases}
$$

where $\mathrm{i}=\mathrm{j}=1$ and $(\mathrm{p}, \mathrm{q})$ represent block indices.

\section{PSO Based Parameter Optimization}

Scaling factors, which are used to modify the selected coefficients during watermark embedding, significantly affect the performance of the watermarking scheme. Therefore, the problem of finding suitable scaling factors which can give optimum performance in terms of visual quality and robustness can be viewed as an optimization problem. The optimization problem can be formulated as follows:

\section{Maximize $\mathrm{R}\left(\left[\beta_{\mathrm{k}}\right]\right)$.}

Maintaining $\mathrm{T}\left(\left[\beta_{\mathrm{k}}\right]\right) \geq \mathrm{T}_{\mathrm{th}}$.

where $\mathrm{R}($.$) and \mathrm{T}($.$) are robustness and imperceptibility$ achieved using the scaling factors $\beta_{\mathrm{k}}$ respectively, and $\mathrm{T}_{\mathrm{th}}$ is user defining quality threshold value. Fitness function
(FF), through which the optimization problem is guided toward optimum performance, should be formulated properly putting these watermarking objectives into consideration.

In this paper, population based stochastic optimization technique PSO is employed to find a solution to the problem. The algorithm was first developed by Eberhart and Kennedy in 1995 [14]. Randomly generating a swarm of particles from search space having initial position $\mathrm{x}_{\mathrm{i}}(\mathrm{t})$ and velocity $\mathrm{v}_{\mathrm{i}}(\mathrm{t})$ is the first step in a PSO algorithm. The quality of each particle is evaluated using formulated fitness function.

$$
F F=T\left(\beta_{k}\right)+\gamma \sum_{i=1}^{N} R_{i}\left(\beta_{k}\right)
$$

Where $R_{i}$ is robustness measured against $i^{\text {th }}$ attack, $T$ is quality achieved, and $\mathrm{N}$ is the total number of attacks performed. $\gamma$ is a weighting factor for $\mathrm{R}_{\mathrm{i}}$.

Based on the fitness value, each particle personal best $\left(\mathrm{P}_{\text {best }}\right)$ and overall global best $\left(\mathrm{g}_{\text {best }}\right)$ are determined. Unless predefined stopping conditions are satisfied, the particles keep on moving around the searching space looking for optimum values and update their velocity and position in every hop using equation (14) and (15) respectively.

$$
\begin{gathered}
v_{i}(t+1)=W v_{i}(t)+C_{1} \operatorname{rand}\left(p_{\text {best }}-x_{i}(t)\right)+C_{2} \operatorname{rand}\left(g_{\text {best }}-x_{i}(t)\right) \\
x_{i}(t+1)=x_{i}(t)+v_{i}(t+1)
\end{gathered}
$$

where $\mathrm{W}$ is inertia weight which determines the step size, $\mathrm{C}_{1}$ and $\mathrm{C}_{2}$ are learning factors which determine the effectiveness of local and global learning. The term rand refers to an operation which randomly generates numbers between 0 and 1. In Fig. 2 the flow chart of PSO algorithm is shown.

We have used the metric peak signal to noise ratio (PSNR) and bit correct rate (BCR) to measure the level of imperceptibility and robustness respectively, and their definition is given in section VI. The overall steps for PSO based parameter optimization are summarized and given as follows:

i. Set suitable initial values for parameters (i.e., initialize particles).

ii. Use the parameters and embed a binary watermark into host image following the procedure which is given in subsection A.

iii. Perform different attacks on watermarked image.

iv. Extract watermark from attacked image using the procedure given in sub-section $B$.

v. Compute fitness value and obtain $\mathrm{p}_{\text {best }}$ and $\mathrm{g}_{\text {best }}$.

vi. Compute next velocity and position of each particle using equation (13) and (14).

vii. Update current $\mathrm{p}_{\text {best }}, \mathrm{g}_{\text {best, }}$ velocity and position.

viii. Repeat step ii to vii until stopping conditions are satisfied.

Finally, use obtained optimum parameters $\left(\beta_{k}\right)$ and embed a watermark 


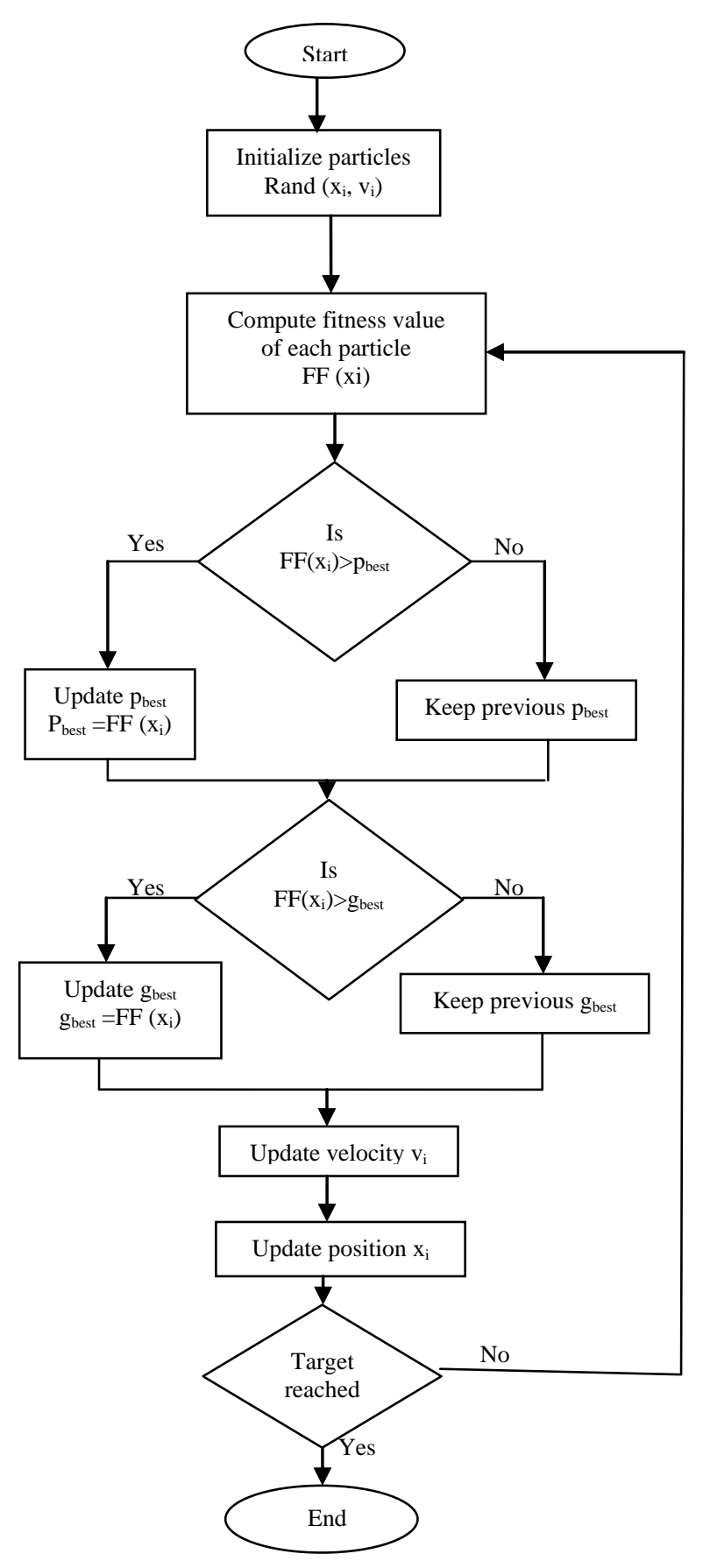

Fig.2. Flow chart for PSO algorithm.

\section{PROPOSED SCHEME}

In this section, the performance of the proposed scheme is evaluated through simulation using eight standard grayscale images of size $512 \times 512$ as host image. A logo of copyright of size $32 \times 32$ is used as a watermark image. The host images and the watermark logo are shown in Fig. 3.

The watermark is embedded into the host images using proposed algorithm, and the level of quality distortion caused due to watermark insertion is evaluated using a PSNR metric. For watermarked image $I_{w}$ and host image $\mathrm{I}_{\mathrm{G}}$ of size $\mathrm{M} \times \mathrm{N}$, the PSNR is computed by comparing the two images as follows.

$$
P S N R=10 \log \frac{255^{2}}{1 / M N \sum_{i=1}^{M} \sum_{j=1}^{N}\left(I_{w}(i, j)-I_{G}(i, j)\right)^{2}} d B
$$

The watermarked images are shown in fig. 4, and the corresponding PSNR values, which show the quality achieved, are presented in Table 1 .

The visual quality of watermarked images in fig. 4 and the PSNR values in Table 1 confirm that the proposed watermarking algorithm can achieve higher imperceptibility. When good imperceptibility achieved, watermarked image appears nearly identical to the original host image and has high PSNR value. Generally, imperceptibility within $38 \mathrm{~dB}$ is the minimum accepted value of PSNR [15].

Different image processing techniques, which are performed on watermarked images aiming to manipulate or destroy the embedded watermark information, can be considered as attacks. In this work, to evaluate the level of robustness, we have performed numerous attacks on watermarked image using a Matlab image processing toolbox. The attacks are: Gaussian white noise $(\mu=0$ and $\mathrm{v}=0.001)$, Salt \& pepper noise $(\mathrm{D}=0.002)$, Median filter (3x3), Histogram equalization, Gamma correction $(\gamma=0.925)$, Cropping ( $25 \%$ of image portion), JPEG compression, Average filter $(3 \times 3)$, Gaussian low pass filter $(3 \times 3)$, Camera motion $($ len $=9, \theta=0)$, Bit plane removing (i.e., LSB image discarded), Image sharpening, Resizing (512 $256 \quad 512)$ and Weiner filter. 


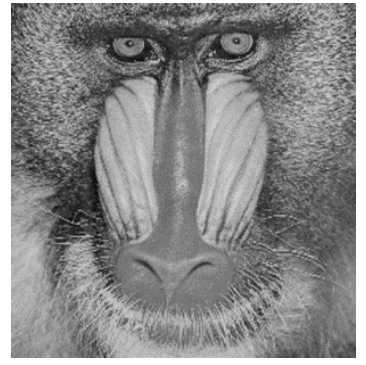

(a)

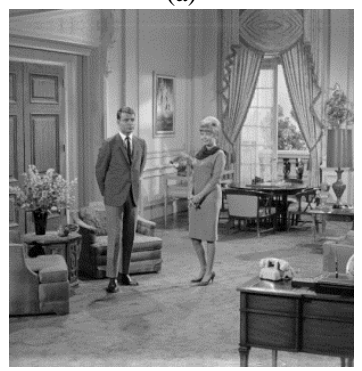

(e)

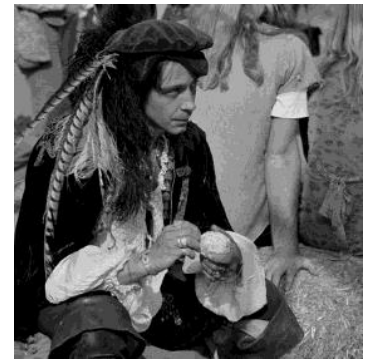

(b)

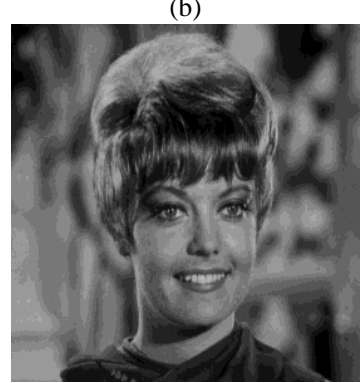

(f)

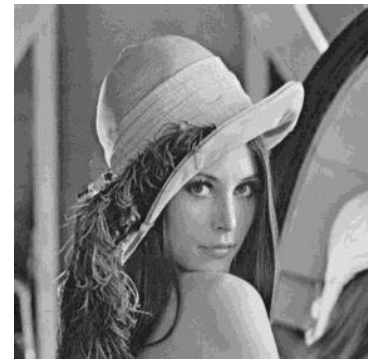

(c)

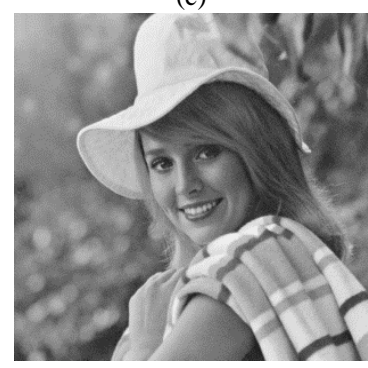

(g)

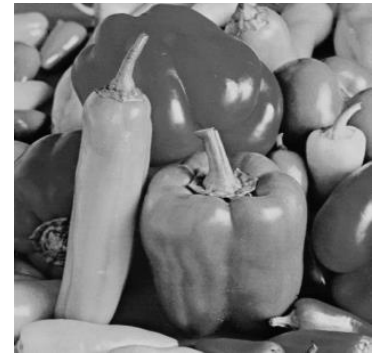

(d)

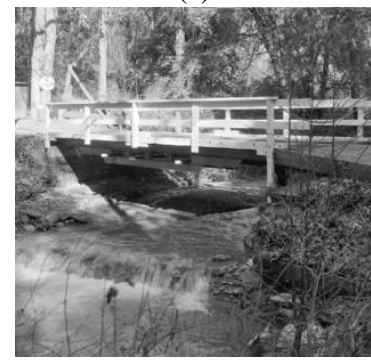

(h)

(C)

(i)

Fig.3. The host images and the watermark logo: (a) Baboon, (b) Pirate, (c) Lena, (d) peppers, (e) living room, (f) Zelda, (g) Elaine, (h) Bridge, (i) watermark logo

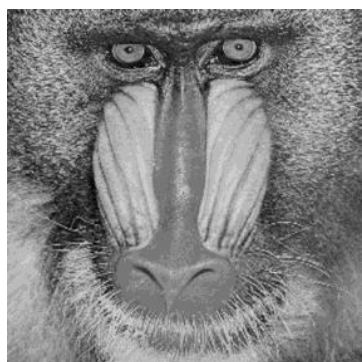

(a)

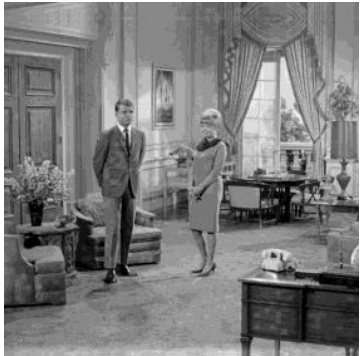

(e)

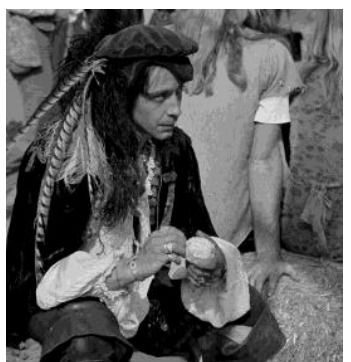

(b)

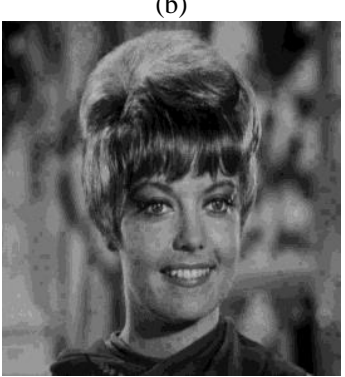

(f)

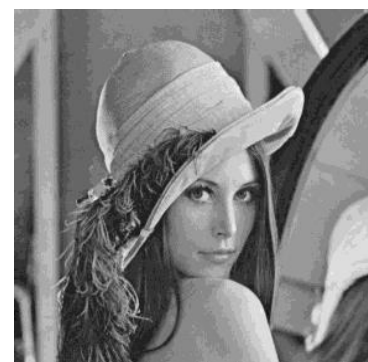

(c)

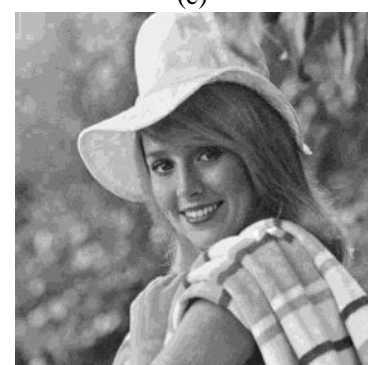

(g)

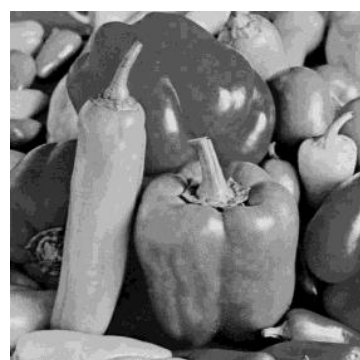

(d)

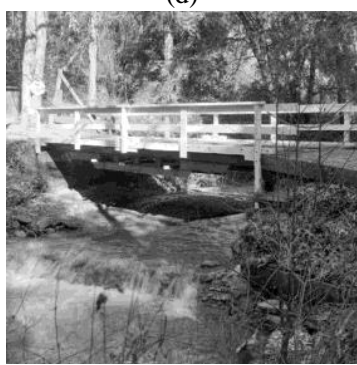

(h)

Fig.4. Watermarked images: (a) Baboon, (b) Pirate, (c) Lena, (d) peppers, (e) living room, (f) Zelda, (g) Elaine, (h) Bridge

Table 1 . The quality that can be achieved, for each host image, in terms of PSNR (dB).

\begin{tabular}{|c|c|c|c|c|c|c|c|c|}
\hline Host Images & Baboon & Pirate & Lena & Peppers & Living room & Zelda & Elaine & Bridge \\
\hline Quality in PSNR (dB) & 44.2586 & 44.4857 & 47.4493 & 43.7711 & 44.1419 & 44.2342 & 43.2457 & 43.1219 \\
\hline
\end{tabular}

For the sake of convenience, in the later sections, the attacks are identified as GN, SPN, MF, HE, GC, CRP, JPEG-C, AVF, G-LPF, CM, BP, SRP, RS, and WF respectively. In fig. 5 and fig. 6 attacked watermarked Lena images are shown.
The presence of the embedded watermark withstanding applied attacks is verified by extracting the watermark from the attacked image using the proposed extracting procedure. 


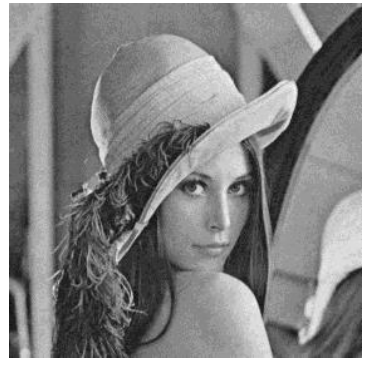

(a)

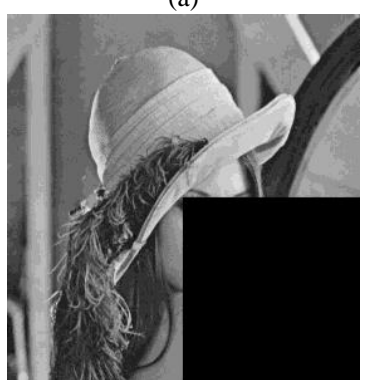

(e)

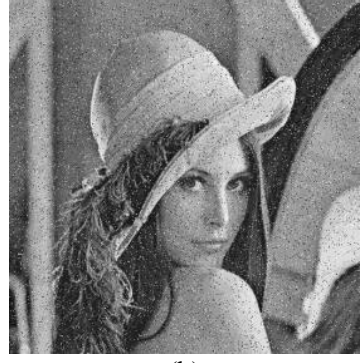

(b)

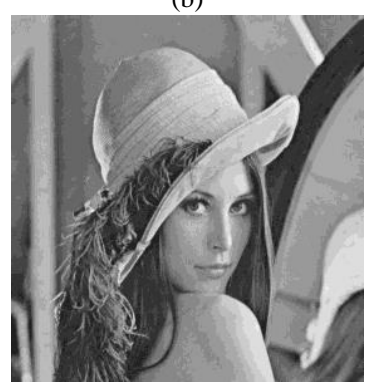

(f)

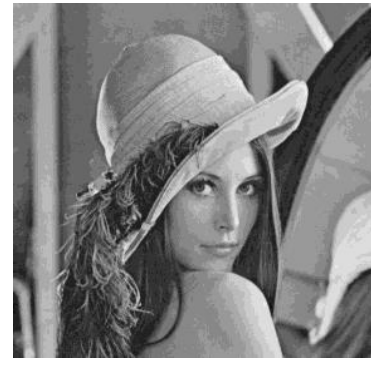

(c)

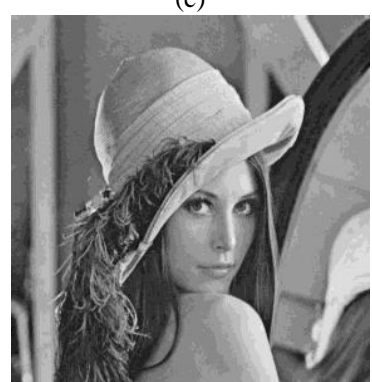

(g)

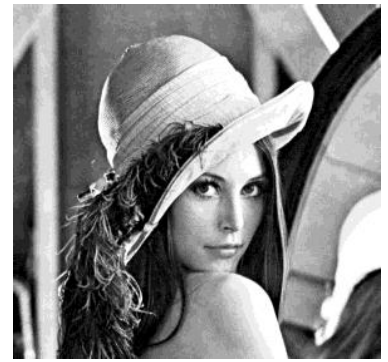

(d)

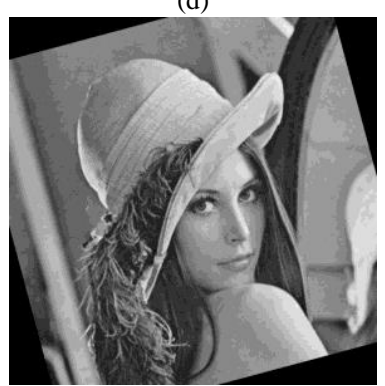

(h)

Fig.5.Attacked watermarked Lena image: (a) Gaussian noise, (b) Salt \& pepper noise, (c) Median filtering, (d) Histogram Equalization, (e) Cropping, (f) Contrast adjusting, (g) JPEG compression, (h) Rotation.

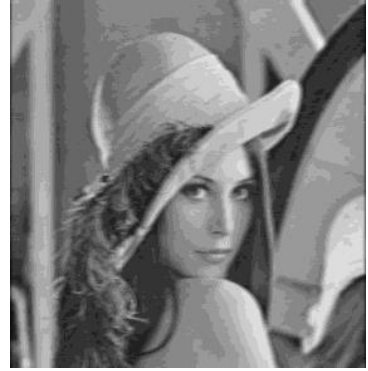

(i)

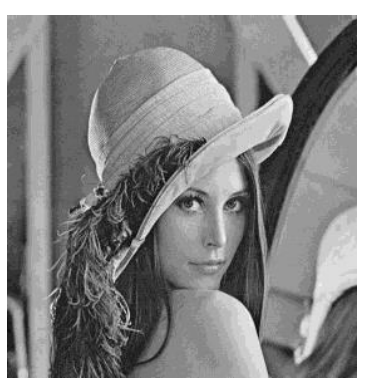

(j)

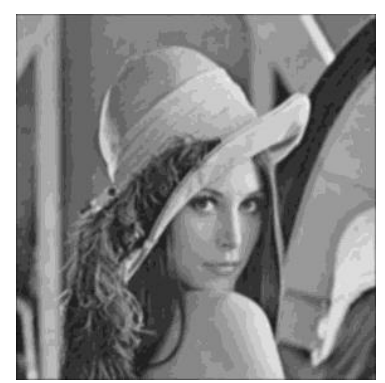

(k)

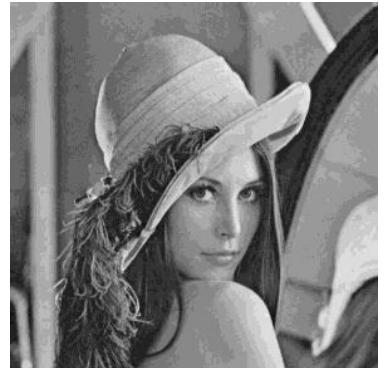

(1)

Fig. 6.Attacked watermarked Lena image: (i) Wiener filter, (j) Image sharpening, (k) Average filter, (l) Bit plane replacement.

Bit Correct Rate (BCR) is employed to compute the degree of similarity between the original and extracted watermark images. For original watermark $\mathrm{W}_{\mathrm{b}}(\mathrm{i}, \mathrm{j})$ and extracted watermark $\mathrm{W}_{\mathrm{E}}(\mathrm{i}, \mathrm{j})$, the robustness metric BCR can be computed using the following equation.

$$
B C R=\frac{\sum_{j=1}^{p} \sum_{i=1}^{q} \overline{W_{b}(i, j) \otimes W_{E}(i, j)}}{p q}
$$

where $\otimes$ and $\overline{(.)}$ are binary Xor and Not operator respectively. In fig. 7, extracted watermark images from attacked watermarked Lena and Baboon images are shown. The obtained result illustrate that the proposed method can give good quality extracted watermark image against the all attacks performed except rotation attack. Table 2 shows the BCR values between the original watermark and extracted watermark after the attacks.

\begin{tabular}{|c|c|c|c|}
\hline \multirow[t]{2}{*}{ Attacks } & Extracted watermarks form & \multirow{2}{*}{ Attacks } & Extracted watermarks form \\
\hline & \begin{tabular}{|l|l|} 
Lena & Baboon \\
\end{tabular} & & \\
\hline No attack (NoA) & & $\begin{array}{l}\text { JPEG compression attack QF=65\% } \\
\text { (JPEG-C) }\end{array}$ & \\
\hline Gaussian noise attack (GN) & & Average filter (AVF) & \\
\hline Salt \& pepper noise attack (SPN) & & Gaussian filter (G-LPF) & \\
\hline Median filtering attack (MF) & & Camera motion $(\mathrm{CM})$ & \\
\hline $\begin{array}{l}\text { Histogram Equalization attack } \\
\text { (HE) }\end{array}$ & & Bit plane removing (BP) & \\
\hline
\end{tabular}




\begin{tabular}{|l|l|l|l|l|l|}
\hline Contrast adjusting attack $(\mathrm{CA})$ & (C) & (C) & Image sharpening $(\mathrm{SRP})$ & (C) \\
\hline Cropping attack $(\mathrm{CRP})$ & $\mathbf{( C )}$ & $\mathbf{( C )}$ & Resizing $(\mathrm{RS})$ & (C) \\
\hline Rotation attack $0.5^{\circ}(\mathrm{RT})$ & Wiener filter $(\mathrm{WF})$ & (C) & (C) \\
\hline
\end{tabular}

Fig.7. Extracted watermark images from the attacked watermarked Lena and Baboon images.

When BCR value of extracted watermark is close to 1 under applicable attacks, then the watermarking scheme is said to be robust against these attacks. From Table 2, it can be observed that the maximum BCR value obtained is 1 and a minimum value is 0.5104 which is for rotation attack. Hence, the obtained results highlight the performance of the proposed methods under different attacks. Except for rotation attack, the proposed scheme shows the highest robustness against all other attacks performed. The corresponding optimized multiple scaling factors, which provide the best PSNR and BCR values for Lena, Baboon, and Bridge host images, are presented in Table 3. These are obtained from PSO based watermarking scheme. In order to evaluate the performance level of the proposed method, we have compared our results with other existing methods, which are reported in [16-18] and is shown in Table 4. The BCR results, which are given in the Table 4 , are obtained by calculating the average BCR over the eight host images. It can be seen from the result that the proposed scheme outperformed previous schemes in terms of imperceptibility and robustness. However, the robustness against JPEG compression for QF below 60\% and cropping attack is slightly lower than that of J. Das, et. Al. and J. Wang, et al. respectively. In fact, Higher robustness could come at the expense of some degradation in quality due to the conflict between them.

Table 2. BCR between original watermark and extracted watermark under different attacks.

\begin{tabular}{|c|c|c|c|c|c|c|c|c|}
\hline \multirow[t]{2}{*}{ Attacks } & \multicolumn{8}{|c|}{ Watermarked images } \\
\hline & Lena & Baboon & Pirate & Peppers & Living room & Zelda & Elaine & Bridge \\
\hline NoA & 1 & 1 & 1 & 1 & 1 & 1 & 1 & 1 \\
\hline GN $(\mu=0, v=0.001)$ & 0.9936 & 0.9959 & 0.9851 & 0.9816 & 0.9871 & 0.9735 & 0.9870 & 0.9796 \\
\hline SPN $(D=0.002)$ & 0.9851 & 0.9991 & 0.9766 & 0.9594 & 0.9647 & 0.9583 & 0.9506 & 0.9741 \\
\hline MF (3x3) & 0.9836 & 0.9983 & 0.9712 & 0.9545 & 0.9562 & 0.9372 & 0.9658 & 0.9517 \\
\hline $\mathrm{HE}$ & 0.9872 & 0.9975 & 0.9851 & 0.9578 & 0.9742 & 0.9709 & 0.9718 & 0.9712 \\
\hline $\mathrm{GC}(\gamma=0.925)$ & 0.9751 & 0.9915 & 0.9808 & 0.9739 & 0.9658 & 0.9517 & 0.9704 & 0.9867 \\
\hline CRP $(25 \%)$ & 0.9715 & 0.9987 & 0.9829 & 0.9546 & 0.9742 & 0.9796 & 0.9615 & 0.9684 \\
\hline $\operatorname{RT}\left(0.5^{0}\right)$ & 0.7137 & 0.7345 & 0.7971 & 0.7566 & 0.6278 & 0.5774 & 0.5483 & 0.5104 \\
\hline JPEG-C $(\mathrm{QF}=65 \%)$ & 0.9871 & 0.9809 & 0.9808 & 0.9807 & 0.9729 & 0.9673 & 0.9852 & 0.9824 \\
\hline $\operatorname{AVF}(3 \times 3)$ & 0.9521 & 0.9451 & 0.9396 & 0.9377 & 0.9419 & 0.9218 & 0.9417 & 0.9349 \\
\hline G-LPF (3x3) & 0.9936 & 1 & 0.9845 & 0.9879 & 0.9860 & 0.9787 & 0.9896 & 0.9843 \\
\hline $\mathrm{CM}(\mathrm{len}=9, \theta=0)$ & 0.9942 & 0.9964 & 0.9766 & 0.9882 & 0.9773 & 0.9603 & 0.9637 & 0.9862 \\
\hline BPR(LSB removed) & 0.9945 & 0.9954 & 0.9845 & 0.9867 & 0.9909 & 0.9902 & 0.9934 & 0.9693 \\
\hline SRP & 0.9882 & 0.9845 & 0.9766 & 0.9847 & 0.9878 & 0.9852 & 0.9819 & 0.9793 \\
\hline RS $(50 \%)$ & 0.9766 & 0.9998 & 0.9826 & 0.9853 & 0.9698 & 0.9533 & 0.9863 & 0.9818 \\
\hline WF $(3 \times 3)$ & 0.9875 & 0.9893 & 0.9829 & 0.9856 & 0.9524 & 0.9581 & 0.9813 & 0.9732 \\
\hline
\end{tabular}

Table 3. Optimized multiple scaling factors (MSF) obtained from PSO.

\begin{tabular}{|c|c|c|c|c|c|c|c|}
\hline Cover image & PSNR & \multirow{2}{*}{$\begin{array}{c}\text { BCR (JPEG, } \\
\text { QF=10) }\end{array}$} & \multicolumn{5}{|c|}{ Optimized Scaling factors $\left(\beta_{\mathrm{k}}\right.$ ) for different ranges } \\
\cline { 4 - 7 } & & & $\beta_{1}$ & $\beta_{2}$ & $\beta_{3}$ & $\beta_{4}$ & $\beta_{5}$ \\
\hline Lena & 47.4493 & 0.6521 & 0.0163 & 0.0676 & 0.0721 & 0.0930 & 0.1018 \\
\hline Baboon & 44.2586 & 0.6491 & 0.0297 & 0.0560 & 0.0693 & 0.0810 \\
\hline Bridge & 43.5219 & 0.6573 & 0.0527 & 0.0556 & 0.0646 & 0.0924 & 0.0932 \\
\hline
\end{tabular}


Table 4. Proposed method robustness comparison with other three methods in terms of BCR.

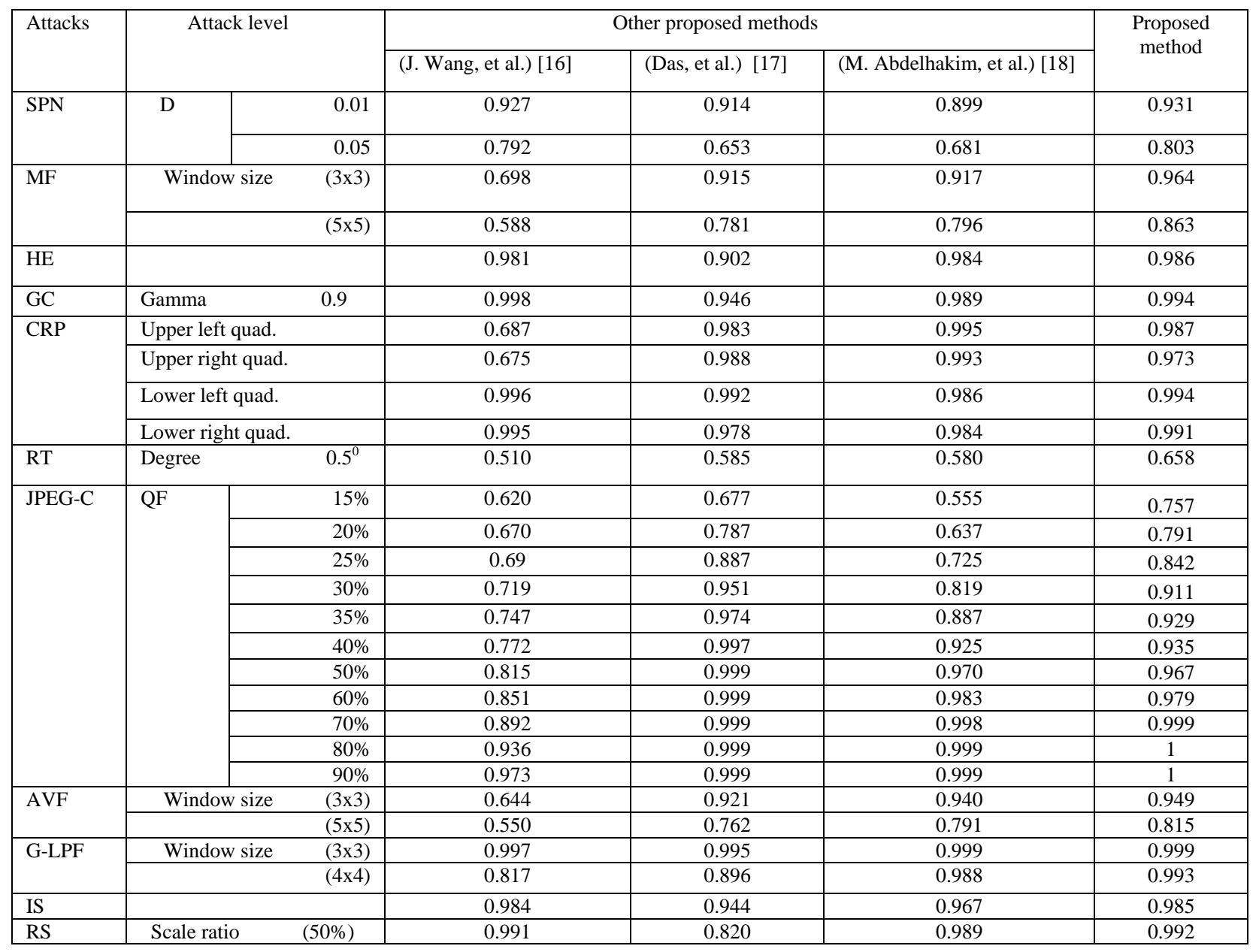

From the overall results presented in this section, we can observe that the new proposed watermarking technique is capable of achieving higher imperceptibility and robustness against both image processing and geometric attacks and is considerably more robust than other three compared methods.

\section{CONCLUSION}

The challenge of designing both robust and imperceptible image watermarking schemes for multimedia copyright protection application gets a solution in this paper, and a new robust image watermarking scheme based on edge detection, SVD and PSO technique that can satisfy the watermarking requirements is presented. To improve the quality of watermarked image the watermark is embedded in selected blocks of the image having large number of edges. Optimized multiple scaling factors are used to modify different range of singular values during watermark embedding process. Different attacks have been performed on watermarked images to evaluate the level of robustness, and the results obtained from the simulation shows that proposed method provides best BCR values for the attacks performed. Three other watermarking techniques have been considered for comparison and the proposed method achieves significantly improved robustness and imperceptibility compared to others.

\section{REFERENCES}

[1] I. J. Cox, Ed., Digital watermarking and steganography, 2nd ed. Amsterdam; Boston: Morgan Kaufmann Publishers, 2008.

[2] Anuja Dixit, Rahul Dixit,"A Review on Digital Image Watermarking Techniques", International Journal of Image, Graphics and Signal Processing(IJIGSP), Vol.9, No.4, pp.56-66, 2017.DOI: 10.5815/ijigsp.2017.04.07

[3] Y. S. Singh, B. P. Devi, and K. M. Singh, "A review of different techniques on digital image watermarking scheme," Int. J. Eng. Res., vol. 2, no. 3, pp. 193-199, 2013.

[4] J. Abraham and V. Paul, "An imperceptible spatial domain color image watermarking scheme," J. King Saud Univ. - Comput. Inf. Sci., Dec. 2016.

[5] M. Prajapati, "Transform Based Digital Image Watermarking Techniques for Image Authentication."

[6] H. B. Kekre, Tanuja Sarode, Shachi Natu,"Performance Comparison of Watermarking Using SVD with Orthogonal Transforms and Their Wavelet Transforms", IJIGSP, vol.7, no.4, pp.1-18, 2015.DOI: 10.5815/ijigsp.2015.04.01

[7] M.-R. Zhang, C.-H. Lu, and K.-C. Yi, "A novel transform-domain image watermark," Can. J. Electr. 
Comput. Eng., vol. 29, no. 3, pp. 179-182, 2004.

[8] D. Y. Lv, G. L. Xu, X. Chen, and Y. P. Tian, "A new watermarking method based on chaotic mixing and SVD," Imaging Sci. J., vol. 60, no. 3, pp. 127-137, Jun. 2012.

[9] R. Liu and T. Tan, "An SVD-based watermarking scheme for protecting rightful ownership," IEEE Trans. Multimed., vol. 4, no. 1, pp. 121-128, 2002.

[10] F. Golshan and K. Mohammadi, "SVD-based digital image watermarking using adaptive generated watermark," The Imaging Science Journal, vol. 62, no. 1, pp. 3-10, Jun. 2013.

[11] Y.-R. Wang, W.-H. Lin, and L. Yang, "An intelligent watermarking method based on particle swarm optimization," Expert Syst. Appl., vol. 38, no. 7, pp. 8024-8029, Jul. 2011.

[12] Chih-Wei Tang and Hsueh-Ming Hang, "A feature-based robust digital image watermarking scheme," IEEE Trans. Signal Process., vol. 51, no. 4, pp. 950-959, Apr. 2003.

[13] Y.-R. Wang, W.-H. Lin, and L. Yang, "A blind PSO watermarking using wavelet trees quantization," in Machine Learning and Cybernetics (ICMLC), 2011 International Conference on, 2011, vol. 4, pp. 1612-1616.

[14] J. Kennedy and R. Eberhart, "Particle swarm optimization," 1995, vol. 4, pp. 1942-1948.

[15] B. E. Khoo, N. M. Makbol, and T. H. Rassem, "Blockbased discrete wavelet transform-singular value decomposition image watermarking scheme using human visual system characteristics," IET Image Process., vol. 10, no. 1, pp. 34-52, Jan. 2016.

[16] J. Wang, H. Peng, and P. Shi, "An optimal image watermarking approach based on a multi-objective genetic algorithm,” Inf. Sci., vol. 181, no. 24, pp. 5501-5514, Dec. 2011.

[17] C. Das, S. Panigrahi, V. K. Sharma, and K. K. Mahapatra, "A novel blind robust image watermarking in DCT domain using inter-block coefficient correlation," AEU Int. J. Electron. Commun., vol. 68, no. 3, pp. 244-253, Mar. 2014.

[18] A. M. Abdelhakim, H. I. Saleh, and A. M. Nassar, "A quality guaranteed robust image watermarking optimization with Artificial Bee Colony," Expert Syst. Appl., vol. 72, pp. 317-326, Apr. 2017.

\section{Authors' Profiles}

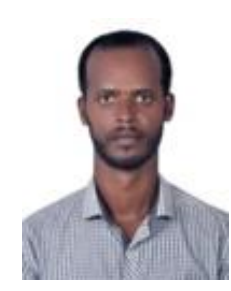

Tamirat Tagesse Takore received his M.Tech in Electronics and Computer Engineering from Addis Ababa University in 2010. He is currently working towards his $\mathrm{Ph} . \mathrm{D}$. at Andhra University College of Engineering, Visakhapatnam, India. His research interests are in the area of image processing and digital signal processing.

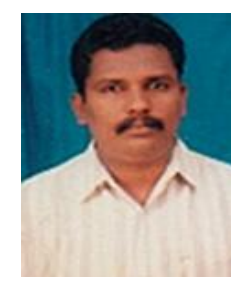

Dr. P. Rajesh Kumar is a professor in Electronics and communication engineering Department, Andhra University College of Engineering, and Visakhapatnam, India. He Received his M.E. and Ph.D. from Andhra University. He has nineteen years experience of teaching undergraduate, postgraduate and guided number of postgraduate theses. He has published forty five research papers in National and International Journals. Presently he is guiding ten Ph.D. students and his research interests are in the area of Digital image processing, Digital signal processing and Radar signal processing.

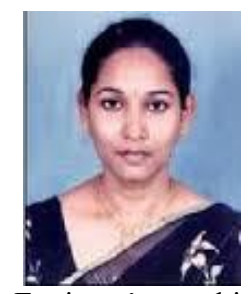

Dr. G. Lavanya Devi is an Ass. Professor in Department of Computer Science and System Engineering Department, Andhra University College of Engineering, Visakhapatnam, India. She has ten years Experience of teaching undergraduate, postgraduate and guided a number of postgraduate theses. She received 'young Engineer' award in 2011 in the field of Computer Science from Institute of Engineers (India). She has published fourteen research papers in both National and International Journals. Her research interest includes, Image processing, Pattern recognition, Fuzzy theory, Data mining, Bioinformatics, Database systems, Network.

How to cite this paper: Tamirat Tagesse Takore, P. Rajesh Kumar, G. Lavanya Devi,"Robust Image Watermarking Scheme Using Population-Based Stochastic Optimization Technique", International Journal of Image, Graphics and Signal Processing(IJIGSP), Vol.9, No.7, pp.55-65, 2017.DOI: 10.5815/ijigsp.2017.07.06 